\title{
The hypoxia marker CAIX is prognostic in the UK phase III VorteX-Biobank cohort: an important resource for translational research in soft tissue sarcoma
}

Laura Forker ${ }^{1}$, Piers Gaunt ${ }^{2}$, Stefano Sioletic ${ }^{3}$, Patrick Shenjere ${ }^{4}$, Robert Potter ${ }^{1}$, Darren Roberts ${ }^{1}$, Joely Irlam $^{1}$, Helen Valentine ${ }^{1}$, David Hughes ${ }^{5}$, Ana Hughes ${ }^{2}$, Lucinda Billingham², Rob Grimer ${ }^{6}$, Beatrice Seddon ${ }^{7}$, Ananya Choudhury ${ }^{1}$, Martin Robinson ${ }^{8}$ and Catharine M L West ${ }^{\star, 1}$

${ }^{1}$ Translational Radiobiology Group, Division of Cancer Sciences, University of Manchester, Manchester Academic Health Science Centre, Christie Hospital NHS Foundation Trust, Wilmslow Road, Manchester M20 4BX, UK; ${ }^{2}$ Cancer Research UK Clinical Trials Unit, Institute of Cancer and Genomic Sciences, University of Birmingham, Edgbaston, Birmingham B15 2TT, UK; ${ }^{3}$ Department of Pathology, Ospedale S.Camillo de Lellis, Rieti 02100, Italy; ${ }^{4}$ Department of Histopathology, The Christie NHS Foundation Trust, Wilmslow Road, Manchester M20 4BX, UK; ${ }^{5}$ Department of Histopathology, Sheffield Teaching Hospitals NHS Trust, Weston Park Hospital, Whitham Road, Sheffield S10 2SJ, UK; ${ }^{6}$ Department of Orthopaedic Oncology, Royal Orthopaedic Hospital NHS Foundation Trust, Bristol Road South, Northfield, Birmingham B31 2AP, UK; ${ }^{7}$ Department of Oncology, University College London Hospitals NHS Foundation Trust, 1st Floor Central, 250 Euston Road, London NW1 2PG, UK and ${ }^{8}$ Department of Oncology, Academic Unit of Clinical Oncology (Cancer Clinical Trials Centre), Weston Park Hospital, Whitham Road, Sheffield S10 2SJ, UK

Background: Despite high metastasis rates, adjuvant/neoadjuvant systemic therapy for localised soft tissue sarcoma (STS) is not used routinely. Progress requires tailoring therapy to features of tumour biology, which need exploration in well-documented cohorts. Hypoxia has been linked to metastasis in STS and is targetable. This study evaluated hypoxia prognostic markers in the phase III adjuvant radiotherapy VorteX trial.

Methods: Formalin-fixed paraffin-embedded tumour biopsies, fresh tumour/normal tissue and blood were collected before radiotherapy. Immunohistochemistry for HIF-1 $\alpha$, CAIX and GLUT1 was performed on tissue microarrays and assessed by two scorers (one pathologist). Prognostic analysis of disease-free survival (DFS) used Kaplan-Meier and Cox regression.

Results: Biobank and outcome data were available for 203 out of 216 randomised patients. High CAIX expression was associated with worse DFS (hazard ratio 2.28, 95\% confidence interval: 1.44-3.59, $P<0.001$ ). Hypoxia-inducible factor- $1 \alpha$ and GLUT1 were not prognostic. Carbonic anhydrase IX remained prognostic in multivariable analysis.

Conclusions: The VorteX-Biobank contains tissue with linked outcome data and is an important resource for research. This study confirms hypoxia is linked to poor prognosis in STS and suggests that CAIX may be the best known marker. However, overlap between single marker positivity was poor and future work will develop an STS hypoxia gene signature to account for tumour heterogeneity. 
Soft tissue sarcomas (STS) are cancers of mesenchymal origin that are relatively rare, with $\sim 3000$ cases per year diagnosed in adults in the United Kingdom (Francis et al, 2013). They display considerable heterogeneity; there are over 50 different malignant histologic subtypes (Fletcher et al, 2013) and can occur in any anatomical position (Clark et al, 2005). Most patients present with localised disease that can be managed with curative intent. A combination of surgery and radiotherapy results in high local control rates (Zagars et al, 2003). However, high-risk patients (high grade, deep, large tumours) have $\sim 50 \% 5$-year survival (Weitz et al, 2003). Most deaths are due to distant metastasis. The use of chemotherapy in the adjuvant/neoadjuvant setting is controversial, due to lack of a consistent overall survival benefit in clinical trials (Pervaiz et al, 2008; Le Cesne et al, 2014). There is an important unmet clinical need to explore new strategies to prevent metastatic spread.

The failure of previous adjuvant/neoadjuvant trials may reflect the molecular heterogeneity of the disease. Progress requires identification of adverse features of tumour biology associated with metastasis that can be targeted with novel systemic agents. VorteX is a phase III randomised controlled trial assessing whether reduced adjuvant radiotherapy volume can improve limb function in adults with extremity STS. The VorteX-Biobank collected a range of tissue from this well-documented cohort and provides a resource to identify prognostic and predictive biomarkers for novel treatments.

Tumour hypoxia can drive metastasis (Sullivan and Graham, 2007) and has been associated with risk of distant metastasis in STS (Brizel et al, 1996). This is an attractive feature of tumour biology to manipulate, as it can be present across multiple subtypes despite molecular heterogeneity and there are multiple drugs in existence that can target hypoxic cell populations (Wilson and Hay, 2011). Some of these can reduce lung metastases in preclinical models in the adjuvant/neoadjuvant setting (Lunt et al, 2010; Liapis et al, 2015). A robust biomarker of tumour hypoxia in STS could potentially identify around half of patients within the current highrisk group who may benefit from hypoxia targeted therapy. Direct measurement of hypoxia using electrodes is impractical and imaging methods are unproven and not used in routine clinical practice (Hammond et al, 2014; Fleming et al, 2015). Previous studies exploring the link between endogenous hypoxia markers (proteins known to be expressed under hypoxia) have yielded conflicting results (Måseide et al, 2004; Shintani et al, 2006; Hoki et al, 2007; Huang et al, 2010; Smeland et al, 2012; Kim et al, 2015) and were limited by low numbers and old samples. This study evaluates the prognostic value of the endogenous markers HIF- $1 \alpha$, CAIX and GLUT1 in a large, modern (2013) phase III trial cohort treated with current surgical and radiotherapy techniques (VorteXBiobank). REMARK guidelines for biomarker studies were followed (McShane et al, 2005) and manual vs automated scoring was compared.

\section{MATERIALS AND METHODS}

Patients and samples. Prospective samples were collected for the VorteX Biobank from consenting adult patients with localised, extremity soft tissue sarcoma receiving surgery with adjuvant radiotherapy as part of the phase III randomised controlled VorteX trial. The study had appropriate ethical approval (LREC 06/MRE/ 03/3) and informed consent was obtained for sample collection and analysis. Fresh tumour, matched normal tissue, formalin-fixed, paraffin-embedded (FFPE) tissue and peripheral blood samples were collected prior to radiotherapy.

Construction of tissue microarrays. Tumour areas in FFPE material were demarcated by the VorteX trial histopathologist
(DH) and $1 \mathrm{~mm}$ diameter cores were taken in triplicate from different areas. A maximum of 120 cores were placed within a single FFPE block in a standardised pattern (MTA-1; Beecher Instruments, Silver Spring, MD, USA). Eleven tissue microarrays (TMAs) were prepared in total.

Immunohistochemistry. Sections were prepared in duplicate from each TMA for staining for a marker of interest and matched negative control. Positive controls included FFPE sections of hypoxic and normoxic cell pellets and tissue sections from other tumours that had shown high or low expression of the markers in previous experiments (Hunter et al, 2014). Tumour capillary staining was used as an additional internal control for GLUT1 staining.

Hypoxia-inducible factor- $1 \alpha$ and CAIX staining was performed using the Bond-Max Automated staining system (Leica Biosystems, Milton Keynes, UK). Slides were dewaxed and rehydrated before antigen retrieval at $\mathrm{pH} 9.0$ for $40 \mathrm{~min}$ at $100{ }^{\circ} \mathrm{C}$. Three per cent hydrogen peroxide solution was used to block endogenous peroxidases. For HIF- $1 \alpha$ the primary antibody was mouse monoclonal HIF-1 $\alpha$ (BD Biosciences, Oxford, UK; 610959) (1:50 dilution) and the negative control was mouse IgG1 (Dako, Ely, UK; X0931). For CAIX the primary antibody was mouse monoclonal NCL-L-CAIX (Novacastra, Leica Biosystems, Milton Keynes, UK) ( $1: 100$ dilution) and the negative control was mouse IgG2a (Dako; X0943). All dilutions were in antibody diluent (Leica; AR9352) and negative controls were diluted to the same protein concentration as the primary. Slides were incubated for $8 \mathrm{~min}$ at room temperature with postprimary rabbit anti-mouse link reagent (Bond Polymer Refine Detection System; Leica; DS9800) and then for a further $8 \mathrm{~min}$ with anti-rabbit polymer-HRP detection reagent (Bond Polymer Refine Detection System; Leica). 3,3'-Diaminobenzidine tetrahydrochloride was applied for $10 \mathrm{~min}$ at room temperature. Slides were then counterstained with haematoxylin.

Glucose transporter 1 staining was performed manually. Slides were dewaxed and rehydrated. Three per cent hydrogen peroxide solution was used to block endogenous peroxidase activity and casein (Vector, Peterborough, UK; SP5020) was used as a protein block. Primary antibody (rabbit polyclonal anti-GLUT1; Alpha Diagnostic International, Source Bioscience, Nottingham, UK; GT$12 \mathrm{~A} 10 \mu \mathrm{g} \mathrm{ml}^{-1}$ ) or negative control (rabbit IgG Vector I-1000 $10 \mu \mathrm{g} \mathrm{ml}^{-1}$ ) was incubated with the slides for $1 \mathrm{~h}$ at $37^{\circ} \mathrm{C}$. Slides were then incubated with secondary antibody (Rabbit Envision Plus HRP Kit; Dako; K4010) for $30 \mathrm{~min}$ at room temperature. $\mathrm{DAB}+(20 \mu \mathrm{l}$ chromogen to $1 \mathrm{ml}$ substrate $)$ was applied for $5 \mathrm{~min}$ at room temperature. Slides were counterstained with haematoxylin for $1 \mathrm{~min}$.

Manual scoring of immunohistochemistry markers. Slides were viewed using Leica SCN400 Image Viewer and scored at $\times 8$ magnification. The percentage of tumour cells per core expressing each marker was determined. Intensity was recorded for potential future use, but was not used in the current analysis in favour of a simpler scoring system. Negative controls were available for comparison. For HIF- $1 \alpha$ only nuclear staining was considered, for CAIX only membrane staining was scored and for GLUT1 membrane and cytoplasmic staining were included.

Cores were scored twice by the same scorer (LF) on different days. For HIF- $1 \alpha$ and GLUT1 all cores were also scored by a specialist sarcoma consultant histopathologist (SS) and for CAIX this was done for all cores with staining present (PS). Scorers were blinded to clinical outcomes. The score of the consultant histopathologist was taken if scores were discordant. The final score was an average across all cores for each tumour type. Definitions of marker positivity were selected to reflect those used in previous publications. 
Automated image analysis for scoring of immunohistochemistry markers. Tissue microarray images were imported into Definiens tissue studio v.4.2 (Definiens, Munich, Germany) and each core labelled with a coordinate according to location. Scoring algorithms were generated and iteratively improved for each marker with quantifiably expressed instructions for tissue detection, nuclear detection, cell stimulation and scoring thresholds (Supplementary Figure 1).

Statistical analysis. The trial primary outcome measures were limb functionality and time to local recurrence, whereas secondary outcomes were disease-free survival (DFS) and overall survival (OS). The clinical outcome measure for the prognostic analysis was DFS, defined as the time from randomisation to local recurrence, metastasis or death. Patients without a DFS event were censored at the date last known to be alive and event free. Estimates were calculated using Kaplan-Meier (KM) analysis. The log-rank test was used to determine any difference in the outcome for each biomarker split combination. Hazard ratios (HRs) and 95\% confidence intervals (CIs) were obtained using Cox regression analysis (univariable and multivariable). Univariable Cox modelling was used to identify clinicopathologic factors correlated to DFS in this cohort including grade, size (combined as stage), depth, gender, surgical margin, tumour location (upper or lower limb), WHO performance status and age. Factors significant at the 5\% level in the univariable analysis were included in multivariable analyses with each of the study biomarkers. Spearman's correlation and Bland-Altman (Bland and Altman, 1986) plots were used to assess intra- and interobserver variability for duplicate and independent scores per core and to compare manual and automated scores per core. Analysis was performed using Stata v.14 (College Station, TX, USA).

\section{RESULTS}

Available material. Between 2007 and 2013, 319 patients were registered. Of these, 216 patients met the eligibility criteria and were randomised between two different volumes of adjuvant radiotherapy. At the time of analysis median follow-up using reverse KM methodology was 5.2 years and event rates were $12 \%$ (25 out of 216) and 40\% (86 out of 216) for local recurrence and any DFS event, respectively. Two hundred and three of 216 randomised patients (with complete clinical outcome data) consented to the VorteX-Biobank. Table 1 summarises the baseline characteristics in the Biobank population. Tissue collected is summarised in Supplementary Table 1. Staining results and clinical outcome data were available for 165, 183 and 179 patients for HIF$1 \alpha$, CAIX and GLUT1, respectively (Figure 1). A scoring result was not available for every patient due to TMA degradation, lack of tumour in core or poor image quality.

Protein marker expression. Supplementary Figure 2 shows staining patterns for the three markers. Hypoxia-inducible factor- $1 \alpha$ staining was clearly nuclear and CAIX clearly membranous, consistent with the expected location for activity of these proteins. Very little additional staining was seen for these markers when compared to the negative control. GLUT1 staining was membranous and cytoplasmic, both were scored as a GLUT1 score based on both membranous and cytoplasmic staining has previously been shown to correlate with oxygen electrode measurements in cervix cancer (Airley et al, 2001). Results and thresholds defining positivity for each marker are included in Supplementary Table 2. The median of all scores was zero for all three markers; therefore, cores with any staining present were considered positive. The median of all results greater than zero was used to define strong positive. Duplicate and independent scores per core correlated well (Spearman's $\rho>0.8$ ) and Bland-Altman
Table 1. Baseline characteristics

$n=203 \%=100$

Age

Median 60 years, interquartile range $48-69$ years

\section{Gender}

\section{Male}

Female

WHO performance status

\begin{tabular}{|c|c|c|}
\hline $\begin{array}{l}0-1 \\
2-3 \\
\text { Unknown }\end{array}$ & $\begin{array}{c}163 \\
5 \\
35\end{array}$ & $\begin{array}{c}80 \\
2 \\
18\end{array}$ \\
\hline \multicolumn{3}{|l|}{ Grade } \\
\hline $\begin{array}{l}1 \\
2 \\
3\end{array}$ & $\begin{array}{c}14 \\
39 \\
150 \\
\end{array}$ & $\begin{array}{c}7 \\
19 \\
74\end{array}$ \\
\hline \multicolumn{3}{|l|}{ Depth } \\
\hline $\begin{array}{l}\text { Superficial } \\
\text { Deep } \\
\text { Unknown }\end{array}$ & $\begin{array}{c}36 \\
166 \\
1\end{array}$ & $\begin{array}{c}18 \\
82 \\
0\end{array}$ \\
\hline \multicolumn{3}{|l|}{ Size } \\
\hline $\begin{array}{l}\text { T1 }(\leqslant 5 \mathrm{~cm}) \\
\text { T2 }(>5 \mathrm{~cm})\end{array}$ & $\begin{array}{c}19 \\
184\end{array}$ & $\begin{array}{c}9 \\
91\end{array}$ \\
\hline
\end{tabular}

$\mathrm{T} 2(>5 \mathrm{~cm})$

\begin{tabular}{l|l}
5 & 2
\end{tabular}

Surgical margin

Intralesional

Marginal

Wide

\section{Histologic subtype}

Dedifferentiated liposarcoma

Extraskeletal myxoid chrondrosarcoma

Leiomyosarcoma

Malignant peripheral nerve sheath tumour

Malignant solitary fibrous tumour

Myxofibrosarcoma

Myxoid liposarcoma

Pleomorphic liposarcoma

Pleomorphic rhabdomyosarcoma

Synovial sarcoma

Undifferentiated pleomorphic sarcoma

Other

Unknown

\begin{tabular}{|c|c|}
\hline 5 & 2 \\
4 & 2 \\
9 & 4 \\
6 & 3 \\
4 & 2 \\
52 & 26 \\
27 & 13 \\
5 & 2 \\
3 & 1 \\
6 & 3 \\
68 & 33 \\
13 & 6 \\
1 & 0 \\
\hline
\end{tabular}

Abbreviation: $\mathrm{WHO}=$ World Health Organisation

analyses comparing scores are included in Supplementary Table and Supplementary Figure 5. There was poor overlap for positivity for markers within the same tumour sample (Supplementary Figure 4).

Prognostic significance. Grade and size (combined as stage) and depth were significant in univariable analyses (Table 2) and incorporated in the multivariable analysis. Gender, surgical margin, tumour location (upper or lower limb), WHO performance status and age were not prognostic in the univariable analysis.

Kaplan-Meier survival estimates for HIF- $1 \alpha$, CAIX and GLUT1 are presented in Figure 2. The $P$-value reported on the figures is from the log-rank test. When patients were split into two groups based on HIF- $1 \alpha$ positivity (median split or $>10 \%$ stained), there was no statistically significant difference in DFS (HR 1.41, 95\% CI: $0.86,2.31, P=0.174$ for median split, HR $1.66,95 \%$ CI: $0.98,2.81$, $P=0.058$ for $>10 \%$ stained). When patients were split into three groups (strong positive, weak positive, negative) strong positive $v s$ negative staining was associated with worse DFS (HR 1.76, 95\% CI: $1.01,3.07, P=0.048)$. This was not significant on multivariable analysis (HR 1.31, 95\% CI: 0.74, 2.32, $P=0.348$ ).

Carbonic anhydrase IX was the strongest prognostic marker and was the only marker to retain prognostic significance in any of the multivariable analysis. Poor DFS was associated with CAIX values 


\section{Registered patients $n=319$}

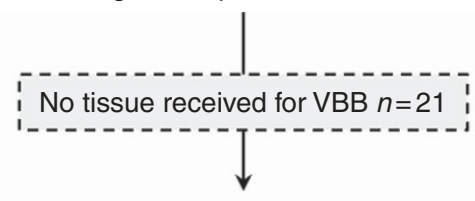

FFPE tissue $n=298$

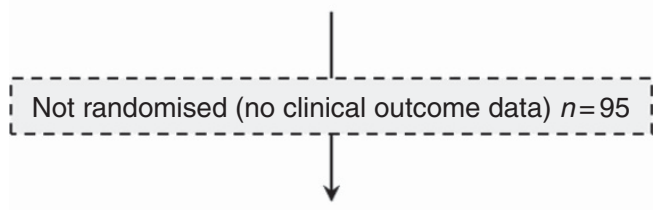

Randomised patients $n=203$

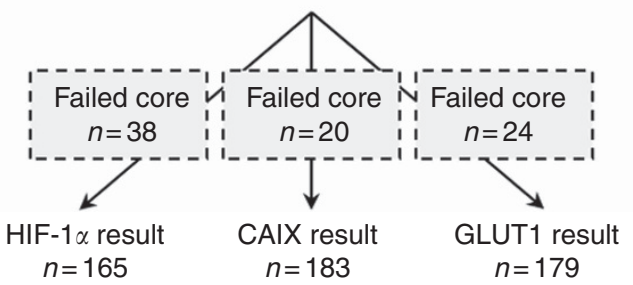

Figure 1. CONSORT diagram. Data for HIF-1 $\alpha$, CAIX and GLUT1 expression were available for 165, 183 and 179 patients randomised in the VorteX trial, respectively. CAIX = carbonic anhydrase IX; FFPE $=$ formalin-fixed, paraffin-embedded; HIF- $1 \alpha=$ hypoxia-inducible factor-1 $\alpha$; GLUT1 = glucose transporter 1; VBB = VorteX-Biobank.

above the median (HR 2.28, 95\% CI: $1.44,3.59, P<0.001$ ) or $>10 \%$ stained (HR 1.75, 95\% CI: 1.04, 2.94, $P=0.037$ ). These estimates remained significant for the median split in the multivariable analyses (HR 2.04, 95\% CI: $1.29,3.25, P=0.002$ ), but not at the $>10 \%$ stained split (HR 1.60, 95\%CI: 0.95, 2.71, $P=0.078$ ). There was no observable difference between strong and weak positive values for CAIX with both being graphically comparable (Figure 2f). There was no difference in baseline characteristics between CAIX-positive and -negative patients (Supplementary Table 4).

Glucose transporter 1 was not prognostic (HR 1.10, 95\% CI: $0.69,1.73, P=0.695$ - median split, HR 1.36, 95\% CI: 0.82, 2.26, $P=0.232->10 \%$ stained, HR 1.39, 95\% CI: 0.80, 2.41, $P=0.238$ - strong positive $v s$ negative).

Comparison of manual and automated scoring. Most cores were successfully scored by automated analysis. Cores were excluded if there was insufficient tissue or core quality was poor. Correlation between manual and automated scores was relatively poor (Spearman's $\rho<0.72$ ) and Bland-Altman analyses comparing scores are included in Supplementary Table and Supplementary Figure 5. KM survival estimates for HIF- $1 \alpha$ and CAIX automated scores are shown in Supplementary Figure 6.

\section{DISCUSSION}

Owing to its rarity and heterogeneity, acquiring adequately sized cohorts of STS patients with both high-quality tumour tissue and robust clinical outcome data is extremely challenging. VorteX is the largest adjuvant radiotherapy trial completed in STS and provides a substantial cohort of patients with high-risk, localised disease covering a range of the more common subtypes seen in adults. The VorteX-Biobank was successful in collecting a wide range of tissue from these patients including FFPE tissue from 301 (94\%) of enrolled patients and 203 (98\%) of randomised patients and matched tumour and normal tissue from 190 (88\%) of randomised patients. This has created a valuable resource for the study of STS
Table 2. Univariable analyses

\begin{tabular}{|c|c|c|}
\hline & HR $(95 \% \mathrm{Cl})$ & $P$-value \\
\hline \multicolumn{3}{|l|}{ Age } \\
\hline & $1.00(0.99,1.02)$ & 0.616 \\
\hline \multicolumn{3}{|l|}{ Gender } \\
\hline Female & 1 & - \\
\hline Male & $1.18(0.76,1.86)$ & 0.460 \\
\hline \multicolumn{3}{|c|}{ WHO performance status } \\
\hline 0 & 1 & \\
\hline 1 & $1.43(0.83,2.48)$ & 0.202 \\
\hline 2 & $1.37(0.33,5.65)$ & 0.661 \\
\hline \multicolumn{3}{|c|}{ Stage (grade, size) } \\
\hline I & $0.46(0.17,1.26)$ & 0.131 \\
\hline II & $0.29(0.15,0.59)$ & 0.001 \\
\hline III & 1 & - \\
\hline \multicolumn{3}{|l|}{ Depth } \\
\hline Deep & 1 & - \\
\hline Superficial & $0.46(0.22,0.95)$ & 0.037 \\
\hline \multicolumn{3}{|c|}{ Surgical margin } \\
\hline Wide & 1 & - \\
\hline Intralesional & $1.15(0.28,4.75)$ & 0.846 \\
\hline Marginal & $1.19(0.77,1.85)$ & 0.439 \\
\hline \multicolumn{3}{|l|}{ Location } \\
\hline Lower limb & 1 & - \\
\hline Upper limb & $0.96(0.77,1.85)$ & 0.898 \\
\hline \multicolumn{3}{|c|}{ HIF-1 $\alpha$ (median split) } \\
\hline Negative & 1 & - \\
\hline Positive & $1.41(0.86,2.31)$ & 0.174 \\
\hline \multicolumn{3}{|c|}{ CAIX (median split) } \\
\hline Negative & 1 & - \\
\hline Positive & $2.28(1.44,3.59)$ & $<0.001$ \\
\hline \multicolumn{3}{|c|}{ GLUT1 (median split) } \\
\hline Negative & 1 & - \\
\hline Positive & $1.10(0.69,1.73)$ & 0.695 \\
\hline
\end{tabular}

biology and identification and validation of biomarkers for novel treatment strategies and highlights the importance of tissue banking alongside clinical trials whenever possible for rare cancers.

Staining patterns for endogenous immunohistochemistry markers of tumour hypoxia were consistent with previous studies in STS (Kim et al, 2015) and other cancers (Airley et al, 2003; Hunter et al, 2014). Spearman's correlation for duplicate and independent scores was high. However, Bland-Altman analyses revealed that while the mean difference between scores was low between independent scores for all markers, the upper and lower limits of agreement were relatively high for CAIX $(-28.9,15.3)$ and GLUT1 ( $-19.7,16.1)$ (Supplementary Figure 3). For CAIX this can be attributed to the fact that only positive cores were second scored for this marker, as due to the quality of the antibody and the staining pattern it was clear if a core was negative. As the optimal definition of positive for CAIX was the presence of any staining, a difference in scores between observers would not alter the classification of the sample if it was used as a biomarker. Although automated scoring would reduce analysis time and the need for specialist pathology input, this was not comparable to manual scoring by a sarcoma pathologist. Correlation between scores and Bland-Altman bias were not within acceptable limits (Supplementary Table and Supplementary Figure 5) and when KM analyses were performed for HIF- $1 \alpha$ and CAIX using automated scores neither were found to be prognostic (Supplementary Figure 6). This is probably due to difficulty in using the software to define regions of stained tumour cells $v s$ stroma or artefact. 
A

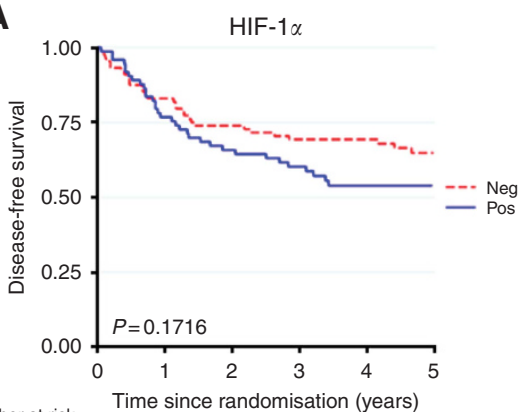

Number at risk Time since randomisation (years)

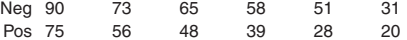

B

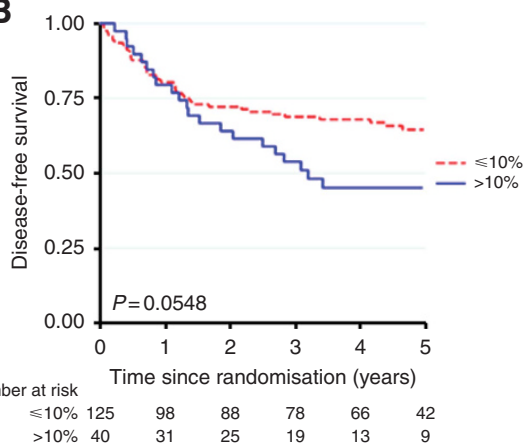

C

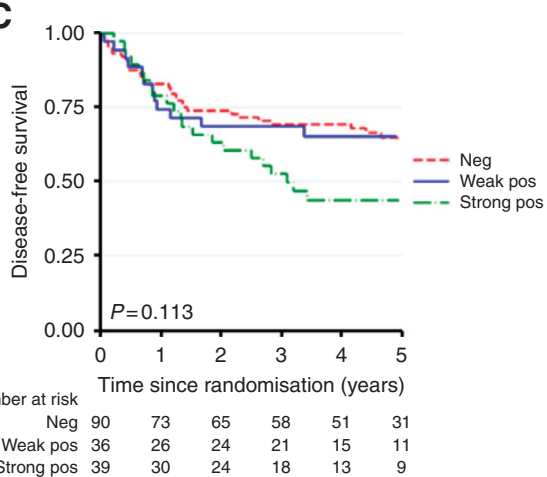

D

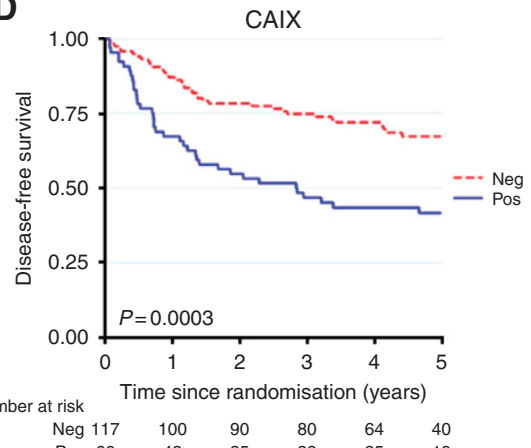

E

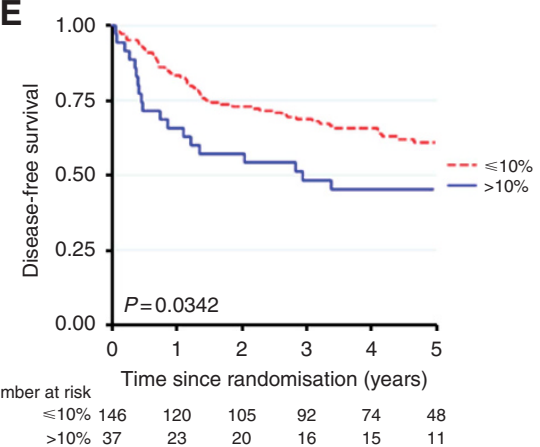

$\mathbf{F}$

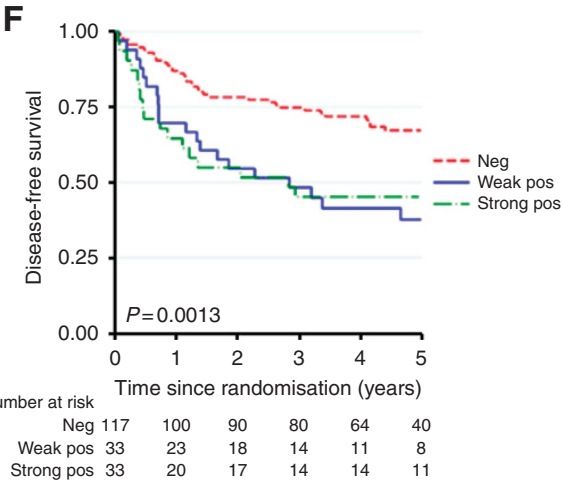

G

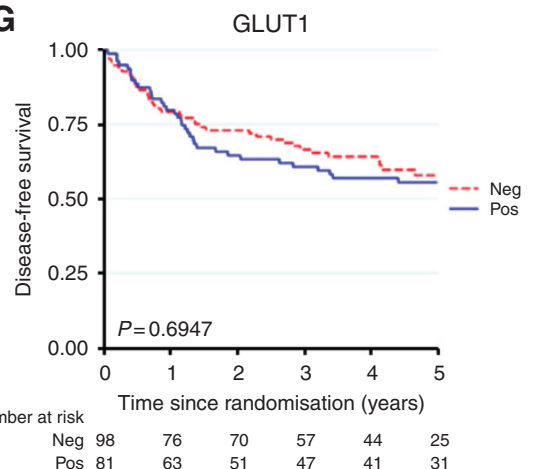

H

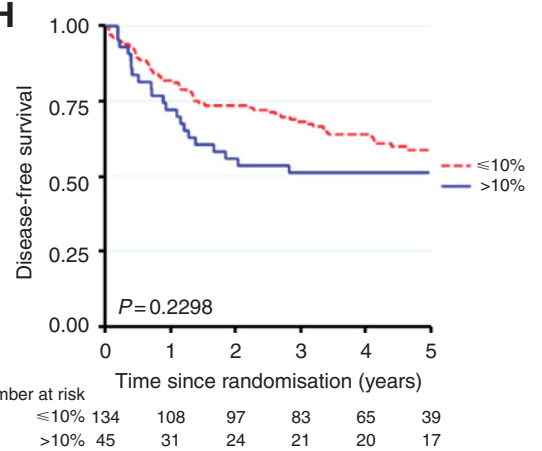

I

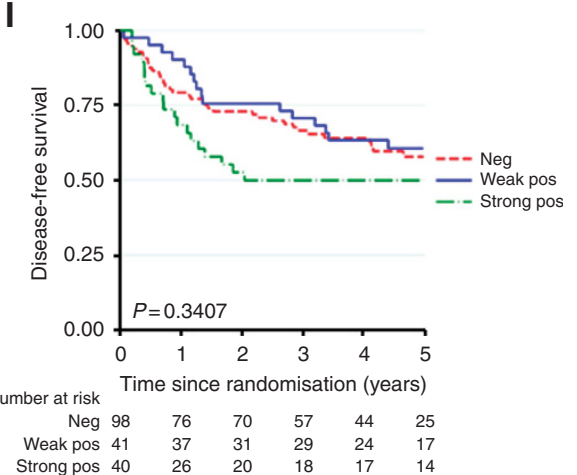

Figure 2. Kaplan-Meier survival estimates for HIF-1 $\alpha$ (A-C), CAIX (D-F) and GLUT1 (G-I). (A) HIF-1 $\alpha$-positive vs -negative, (B) HIF-1 $\alpha \leqslant 10 \%$ vs $>10 \%$, (C) HIF-1 $\alpha$ strong positive, weak positive, negative, (D) CAIX positive vs negative, (E) CAIX $\leqslant 10 \%$ vs $>10 \%$, (F) CAIX strong positive, weak positive, negative, (G) GLUT1 positive vs negative, (H) GLUT1 $\leqslant 10 \%$ vs $>10 \%$ and (I) GLUT1 strong positive, weak positive, negative.

Thresholds for marker positivity were selected to make results comparable with previously published studies. For HIF- $1 \alpha$ this was difficult as previous studies have varied widely from considering $>10 \%$ staining of any intensity as positive (Huang et al, 2010), to defining only tumours with over $50 \%$ of cells strongly stained as positive (Smeland et al, 2012). In the current work, intensity of staining was recorded for potential future use but was not considered at present as a simpler scoring system is likely to be more easily reproducible. For the CAIX studies evaluating membrane staining, one has used a cutoff value of $10 \%$ stained (Kim et al, 2015), and another has defined any staining as positive as it demonstrated no difference in outcome between patients classified as strongly or weakly positive (Måseide et al, 2004). This is consistent with the current work.

Hypoxia-inducible factor- $1 \alpha$ and CAIX expression are associated with worse DFS, albeit CAIX was the only hypoxia marker that was significant in the multivariable analysis. Glucose transporter 1 was not prognostic. Four of five previous studies (Shintani et al, 2006; Hoki et al, 2007; Huang et al, 2010; Smeland et al, 2012; Kim et al, 2015) have associated high HIF-1 $\alpha$ expression with adverse outcomes. Two of three previous studies (Måseide et al, 2004; Smeland et al, 2012; Kim et al, 2015) have associated high CAIX expression with poor outcomes in STS, and high GLUT1 expression has been shown to correlate with worse outcomes in one of two prior studies (Smeland et al, 2012; Kim et al, 2015). Results of the largest previous study (Smeland et al, 2012) ( 200 patients) were almost opposite to the current work with no association for HIF- $1 \alpha$ and CAIX, but prognostic value reported for GLUT1. This may be explained by the fact that the previous study reported different staining patterns that would be considered unusual for the markers in question (mainly cytoplasmic for all markers) and included relatively old samples. The staining patterns scored in the current work are more consistent with the expected location of the active proteins and the cohort is superior to those used in previous studies, as this is a modern phase III trial population (2007-2013) with recent tissue collection and robust outcome data.

Although the current work did not find HIF- $1 \alpha$ staining to be prognostic, it may potentially still be of interest. A recent metaanalysis of 16 studies (5 in STS) evaluating the prognostic 
significance of HIF- $1 \alpha$ in STS and bone sarcomas found high expression was associated with higher rates of metastases, poor OS and poor DFS (Li et al, 2016). A consistent association with poor DFS was found in the STS subgroup analysis, although this only considered 136 patients in three studies. A limitation of HIF- $1 \alpha$ is that it is more difficult to score and define positivity than for CAIX, which can simply be classed as positive in the presence of any membrane staining. Based on this work, CAIX appears to be the best current known hypoxia marker for use on pretreatment biopsies in STS.

Tissue microarrays rather than whole sections were used for this study due to the large number of samples. This is a potential limitation of the work as it assesses a smaller area than a whole section and therefore may be more likely to misclassify a hypoxic tumour as normoxic. However, this was assessed in one of the previous studies of CAIX in STS and TMAs were deemed accurate in determining marker expression provided that at least three cores were taken from two separate areas of tumour (Måseide et al, 2008). Another concern was that there was very limited overlap between expression of the markers in these clinical samples. All three markers are expressed under hypoxia and a degree of coexpression was expected. This may reflect the considerable molecular heterogeneity of STS and indicates that expression of these markers is likely influenced by other pathways. There is therefore a need for a more robust measure of hypoxia in STS than a single protein marker.

Hypoxia-related RNA expression signatures measure changes in multiple genes in response to hypoxia and can account for greater heterogeneity (Harris et al, 2015). Evaluation of a biomarker at the RNA level also reduces inconsistencies in methodology and interpretation between laboratories compared with IHC and is more likely to be reproducible in clinical practice. Hypoxia RNA expression signatures have made the most progress towards use as predictive biomarkers for hypoxia targeted therapy in the clinic, with two signatures (Toustrup et al, 2012; Eustace et al, 2013) currently undergoing prospective validation in phase III trials in head and neck cancer. Recently, one of these signatures has demonstrated prognostic value in a cohort of STS patients (Aggerholm-Pedersen et al, 2016). However, hypoxia signatures generally perform best in the tumour types they were developed for (Eustace et al, 2013) and the signature genes correlated poorly with oxygen electrode measurements in 16 patients indicating that it may not be a good measure of hypoxia in STS (Aggerholm-Pedersen et al, 2016). It may be more appropriate to derive a hypoxia signature specific to STS, as it must be a robust measure of hypoxia if it is to be used as a predictive biomarker for hypoxia targeted therapy. The other tissue available in the VorteX-Biobank is intended to be used for this purpose with the eventual aim of a biomarker-driven trial of hypoxia targeted adjuvant/neoadjuvant therapy in STS.

In summary, evaluation of protein hypoxia markers in a modern phase III clinical trial cohort suggests that CAIX is the best current known hypoxia marker. A simple binary scoring system can easily be implemented to define positivity but still requires manual pathology assessment. However, other measures of hypoxia such as gene signatures may perform better and be more reflective of hypoxia across different subtypes, compensating for greater heterogeneity. Further work is needed to develop a gene signature and confirm that it is a true measure of hypoxia by comparing this to CAIX and other methods of assessing hypoxia (e.g. imaging/ electrodes) before this could be taken forward as a prognostic or predictive biomarker for clinical trials.

\section{ACKNOWLEDGEMENTS}

This work was funded by Cancer Research UK (C1094/A6786) and, for trial results, CRUK/05/003. The work was supported by the Manchester Experimental Cancer Medicine Centre (C1467/ A15578), Cancer Research UK Major Centre and NIHR Manchester Biomedical Research Centre. The study had appropriate ethics approval (LREC 06/MRE/03/3) and informed consent was obtained for sample collection and analysis.

\section{CONFLICT OF INTEREST}

The authors declare no conflict of interest.

\section{REFERENCES}

Aggerholm-Pedersen N, Sorensen BS, Overgaard J, Toustrup K, Baerentzen S, Nielsen OS, Maretty-Kongstad K, Nordsmark M, Alsner J, Safwat A (2016) A prognostic profile of hypoxia-induced genes for localised highgrade soft tissue sarcoma. Br J Cancer 115: 1096-1104.

Airley R, Loncaster J, Davidson S, Bromley M, Roberts S, Patterson A, Hunter R, Stratford I, West C (2001) Glucose transporter Glut-1 expression correlates with tumor hypoxia and predicts metastasis-free survival in advanced carcinoma of the cervix. Clin Cancer Res 7(4): 928-934.

Airley RE, Loncaster J, Raleigh JA, Harris AL, Davidson SE, Hunter RD, West CML, Stratford IJ (2003) GLUT-1 and CAIX as intrinsic markers of hypoxia in carcinoma of the cervix: relationship to pimonidazole binding. Int J Cancer 104(1): 85-91.

Bland MJ, Altman DG (1986) Statistical methods for assessing agreement between two methods of clinical measurement. Lancet 327(8476): 307-310.

Brizel DM, Scully SP, Harrelson JM, Layfield LJ, Bean JM, Prosnitz LR, Dewhirst MW (1996) Tumor oxygenation predicts for the likelihood of distant metastases in human soft tissue sarcoma. Cancer Res 56(5): 941-943.

Clark MA, Fisher C, Judson I, Thomas JM (2005) Soft-Tissue Sarcomas in Adults. N Engl J Med 353(7): 701-711.

Eustace A, Mani N, Span PN, Irlam JJ, Taylor J, Betts GNJ, Denley H, Miller CJ, Homer JJ, Rojas AM, Hoskin PJ, Buffa FM, Harris AL, Kaanders JHAM, West CML (2013) A 26-gene hypoxia signature predicts benefit from hypoxia-modifying therapy in laryngeal cancer but not bladder cancer. Clin Cancer Res 19(17): 4879-4888.

Fleming IN, Manavaki R, Blower PJ, West C, Williams KJ, Harris AL, Domarkas J, Lord S, Baldry C, Gilbert FJ (2015) Imaging tumour hypoxia with positron emission tomography. Br J Cancer 112(2): 238-250.

Fletcher CDM, Bridge JA, Hogendoorn PCW, Mertens F. (eds) (2013) WHO Classification of Tumours of Soft Tissue and Bone. International Agency for Research on Cancer.

Francis M, Dennis N, Charman J, Lawrence G, Grimer R (2013) Bone and soft tissue sarcomas UK incidence and survival: 1996 to 2010.

Hammond EM, Asselin MC, Forster D, O’Connor JPB, Senra JM, Williams KJ (2014) The meaning, measurement and modification of hypoxia in the laboratory and the clinic. Clin Oncol 26(5): 277-288.

Harris BHL, Barberis A, West CML, Buffa FM (2015) Gene expression signatures as biomarkers of tumour hypoxia. Clin Oncol 27(10): 547-560.

Hoki Y, Murata M, Hiraku Y, Ma N, Matsumine A, Uchida A, Kawanishi S (2007) 8-Nitroguanine as a potential biomarker for progression of malignant fibrous histiocytoma, a model of inflammation-related cancer. Oncol Rep 18: 1165-1169.

Huang J-H, Lee FS, Pasha TL, Sammel MD, Karakousis G, Xu G, Fraker D, Zhang PJ (2010) Analysis of HIF-1 $\alpha$ and its regulator, PHD2, in retroperitoneal sarcomas: clinico-pathologic implications. Cancer Biol Ther 9(4): 303-311.

Hunter BA, Eustace A, Irlam JJ, Valentine HR, Denley H, Oguejiofor KK, Swindell R, Hoskin PJ, Choudhury A, West CM (2014) Expression of hypoxia-inducible factor- $1 \alpha$ predicts benefit from hypoxia modification in invasive bladder cancer. Br J Cancer 111(3): 437-443.

Kim JI, Choi KU, Lee IS, Choi YJ, Kim WT, Shin DH, Kim K, Lee JH, Kim JY, Sol MY (2015) Expression of hypoxic markers and their prognostic significance in soft tissue sarcoma. Oncol Lett 9(4): 1699-1706.

Le Cesne A, Ouali M, Leahy MG, Santoro A, Hoekstra HJ, Hohenberger P, Van Coevorden F, Rutkowski P, Van Hoesel R, Verweij J, Bonvalot S, Steward WP, Gronchi A, Hogendoorn PCW, Litiere S, Marreaud S, 
Blay JY, Van Der Graaf WTA (2014) Doxorubicin-based adjuvant chemotherapy in soft tissue sarcoma: pooled analysis of two STBSGEORTC phase III clinical trials. Ann Oncol 25(12): 2425-2432.

Li Y, Zhang W, Li S, Tu C (2016) Prognosis value of hypoxia-inducible factor$1 \alpha$ expression in patients with bone and soft tissue sarcoma: a metaanalysis. SpringerPlus 5(1): 1370.

Liapis V, Labrinidis A, Zinonos I, Hay S, Ponomarev V, Panagopoulos V, DeNichilo M, Ingman W, Atkins GJ, Findlay DM, Zannettino ACW, Evdokiou A (2015) Hypoxia-activated pro-drug TH-302 exhibits potent tumor suppressive activity and cooperates with chemotherapy against osteosarcoma. Cancer Lett 357(1): 160-169.

Lunt SJ, Cawthorne C, Ali M, Telfer BA, Babur M, Smigova A, Julyan PJ, Price PM, Stratford IJ, Bloomer WD, Papadopoulou MV, Williams KJ (2010) The hypoxia-selective cytotoxin NLCQ-1 (NSC 709257) controls metastatic disease when used as an adjuvant to radiotherapy. Br J Cancer 103(2): 201-208.

Måseide K, Kandel RA, Bell RS, Catton CN, O'Sullivan B, Wunder JS, Pintilie M, Hedley D, Hill RP (2004) Carbonic anhydrase IX as a marker for poor prognosis in soft tissue sarcoma. Clin Cancer Res 10(13): 4464-4471.

Måseide K, Pintilie M, Kandel R, Hill RP (2008) Can sparsely and heterogeneously expressed proteins be detected using tissue microarrays? A simulation study of the hypoxia marker carbonic anhydrase IX (CA IX) in human soft tissue sarcoma. Pathol Res Pract 204(3): 175-183.

McShane LM, Altman DG, Sauerbrei W, Taube SE, Gion M, Clark GM (2005) REporting recommendations for tumour MARKer prognostic studies (REMARK). Br J Cancer 93(4): 387-391.

Pervaiz N, Colterjohn N, Farrokhyar F, Tozer R, Figueredo A, Ghert M (2008) A systematic meta-analysis of randomized controlled trials of adjuvant chemotherapy for localized resectable soft-tissue sarcoma. Cancer 113(3): 573-581.
Shintani K, Matsumine A, Kusuzaki K, Matsubara T, Satonaka H, Wakabayashi T, Hoki Y, Uchida A (2006) Expression of hypoxia-inducible factor (HIF)- $1 \alpha$ as a biomarker of outcome in soft-tissue sarcomas. Virchows Archiv 449(6): 673-681.

Smeland E, Kilvaer TK, Sorbye S, Valkov A, Andersen S, Bremnes RM, Busund L-T, Donnem T (2012) Prognostic impacts of hypoxic markers in soft tissue sarcoma. Sarcoma 2012: 541650.

Sullivan R, Graham C (2007) Hypoxia-driven selection of the metastatic phenotype. Cancer Metast Rev 26(2): 319-331.

Toustrup K, Sørensen BS, Lassen P, Wiuf C, Alsner J, Overgaard J (2012) Gene expression classifier predicts for hypoxic modification of radiotherapy with nimorazole in squamous cell carcinomas of the head and neck. Radiother Oncol 102(1): 122-129.

Weitz J, Antonescu CR, Brennan MF (2003) Localized extremity soft tissue sarcoma: improved knowledge with unchanged survival over time. J Clin Oncol 21(14): 2719-2725.

Wilson WR, Hay MP (2011) Targeting hypoxia in cancer therapy. Nat Rev Cancer 11(6): 393-410.

Zagars GK, Ballo MT, Pisters PWT, Pollock RE, Patel SR, Benjamin RS, Evans HL (2003) Prognostic factors for patients with localized soft-tissue sarcoma treated with conservation surgery and radiation therapy. Cancer 97(10): 2530-2543.

(c) (i) This work is licensed under the Creative Commons cc) Attribution 4.0 International License. To view a copy of this license, visit http://creativecommons.org/licenses/by/4.0/

(C) The Author(s) named above 2018

Supplementary Information accompanies this paper on British Journal of Cancer website (http://www.nature.com/bjc) 